\title{
Electron mobility in Si $\delta$-doped GaAs with spatial correlationsin the distribution of charged impurities
}

\section{Citation for published version (APA):}

Shi, J. M., Koenraad, P. M., Stadt, van de, A. F. W., Peeters, F. M., Farias, G. A., Devreese, J. T., Wolter, J. H., \& Wilamowski, Z. (1997). Electron mobility in Si $\delta$-doped GaAs with spatial correlationsin the distribution of charged impurities. Physical Review B: Condensed Matter, 55(19), 13093-13099.

https://doi.org/10.1103/PhysRevB.55.13093

DOI:

10.1103/PhysRevB.55.13093

Document status and date:

Published: 01/01/1997

\section{Document Version:}

Publisher's PDF, also known as Version of Record (includes final page, issue and volume numbers)

\section{Please check the document version of this publication:}

- A submitted manuscript is the version of the article upon submission and before peer-review. There can be important differences between the submitted version and the official published version of record. People interested in the research are advised to contact the author for the final version of the publication, or visit the $\mathrm{DOI}$ to the publisher's website.

- The final author version and the galley proof are versions of the publication after peer review.

- The final published version features the final layout of the paper including the volume, issue and page numbers.

Link to publication

\section{General rights}

Copyright and moral rights for the publications made accessible in the public portal are retained by the authors and/or other copyright owners and it is a condition of accessing publications that users recognise and abide by the legal requirements associated with these rights.

- Users may download and print one copy of any publication from the public portal for the purpose of private study or research.

- You may not further distribute the material or use it for any profit-making activity or commercial gain

- You may freely distribute the URL identifying the publication in the public portal.

If the publication is distributed under the terms of Article 25fa of the Dutch Copyright Act, indicated by the "Taverne" license above, please follow below link for the End User Agreement:

www.tue.nl/taverne

Take down policy

If you believe that this document breaches copyright please contact us at:

openaccess@tue.nl

providing details and we will investigate your claim. 


\title{
Electron mobility in $\mathrm{Si} \delta$-doped GaAs with spatial correlations in the distribution of charged impurities
}

\author{
J. M. Shi \\ COBRA Interuniversity Research Institute, Eindhoven University of Technology, P.O. Box 513, 5600 MB Eindhoven, The Netherlands \\ and Departamento de Física, Universidade Federal do Ceará, Campus do Pici, Caixa Postal 6030, 60455-760 Fortaleza, \\ Ceará, Brazil \\ P. M. Koenraad and A. F. W. van de Stadt \\ COBRA Interuniversity Research Institute, Eindhoven University of Technology, P.O. Box 513, 5600 MB Eindhoven, The Netherlands \\ F. M. Peeters \\ Departement Natuurkunde, Universiteit Antwerpen (UIA), Universiteitsplein 1, B-2610 Antwerpen, Belgium \\ G. A. Farias \\ Departamento de Física, Universidade Federal do Ceará, Campus do Pici, Caixa Postal 6030, 60455-760 Fortaleza, Ceará, Brazil \\ J. T. Devreese \\ Departement Natuurkunde, Universiteit Antwerpen (UIA), Universiteitsplein 1, B-2610 Antwerpen, Belgium \\ and COBRA Interuniversity Research Institute, Eindhoven University of Technology, P.O. Box 513, 5600 MB Eindhoven, \\ The Netherlands \\ J. H. Wolter \\ COBRA Interuniversity Research Institute, Eindhoven University of Technology, P.O. Box 513, 5600 MB Eindhoven, The Netherlands \\ Z. Wilamowski \\ Institute of Physics, Polish Academy of Sciences, Al. Lotników 32/46, 02-668 Warsaw, Poland \\ (Received 3 December 1996)
}

\begin{abstract}
We present a theoretical study of electron mobility in heavily Si $\delta$-doped GaAs in the presence of applied hydrostatic pressure. At low temperature the electron-ionized impurity scattering is the most important scattering mechanism. The presence of $D X$ centers in Si-doped GaAs results in spatial correlations of the charged impurities, which increase the electron mobility through the structure factor of the charged-impurity distribution and/or a decrease in the density of the charged dopants. A Monte Carlo approach has been developed to simulate this distribution in two dimensions for the $d^{+} / D X^{0}$ and $d^{+} / D X^{-}$models. In the mobility calculation, both intrasubband and intersubband scatterings are considered with the electron-electron screening within the random-phase approximation. A detailed comparison between experiment and theory shows that theory excluding the correlation effects underestimates the electron mobility systematically. In cooperation with other mechanisms, e.g., self-compensation of Si dopants, in the $\delta$ layer, both $D X$-center models can explain the experimental results well. This indicates that in order to effectively study the electronic properties of $D X$ centers via the electron mobility in $\delta$-doped structures, the samples must have a relatively low doping concentration in order to prevent self-compensation. [S0163-1829(97)08616-5]
\end{abstract}

\section{INTRODUCTION}

In recent years there has been considerable interest in electron transport properties of $\delta$-doped semiconductors because of their potential applications in high-speed electronics and optoelectronic devices, as well as the fundamental study of the interaction between the electrons and the charged impurities in the limit of strong coupling and the transport properties of systems with several populated subbands. ${ }^{1}$

By now it is well established ${ }^{2}$ that many donors in III-V semiconductors have to be described by the coexistence of a shallow donor state and a deep donor state. Several calculations of electron mobilities, excluding $D X$ centers and as- suming the measured free-electron concentration equal to the doping density, have been performed for $\delta$-doped GaAs structures, and reasonable agreement was obtained with the experimental findings. ${ }^{3,4}$ However, the quantum mobilities measured by Skuras et al..$^{5}$ in $\delta$-doped GaAs structures with a very high doping density $\left(1.1 \times 10^{13} \mathrm{~cm}^{-2}\right)$ in the presence of an external hydrostatic pressure up to $20 \mathrm{kbar}$ cannot be explained by the existing theory described in Refs. 3 and 4 due to population of $D X$ centers. For bulk-doped semiconductors, the influence of $D X$ centers on electron mobility has been investigated both experimentally and theoretically in the presence of a hydrostatic pressure, ${ }^{6-8}$ which shows that $D X$ centers should be negatively charged. However, this 
analysis is valid for the sample having only the effects of $D X$ centers, i.e., the free-electron density is very close to the doping density in the absence of any external hydrostatic pressure; and for $\delta$-doped structures the charge distribution has been studied by both Monte Carlo simulations ${ }^{9}$ and a short-range correlation model. ${ }^{10}$ The experiments have shown that the energy difference between the $D X$ level and the conduction-band minimum is decreased with increasing hydrostatic pressure. This results in a transfer of free electrons to the $D X$ state. As a consequence, the free-electron density will decrease and the electron mobility will be changed. In this paper we will generalize the abovementioned work to investigate theoretically the effects of $D X$ centers on the electron mobility in $\delta$-doped structures.

In the present literature, there are two models describing spatial correlations in the charge distribution of the mixedvalence system which exists when the $D X$ states are populated: the $d^{+} / D X^{0}$ model $^{11}\left(d^{+}+e \rightarrow D X^{0}\right)$, in which the impurities are either positively charged or neutral; and the $d^{+} / D X^{-}$model $^{2}\left(d^{+}+2 e \rightarrow D X^{-}\right)$, in which the impurities are either positively or negatively charged. Since it is still not fully clear which model is applicable, we will discuss both of them in this study of the low-temperature mobility of the electrons. In the actual mobility calculation, both intrasubband and intersubband scatterings are considered with the electron-electron screening within the random-phase approximation (RPA), and the spatial correlations are introduced by the structure factor of the charge distribution in the $\delta$ layer at $T=120 \mathrm{~K}$ since the $(\mathrm{Si}) D X$ centers in $\mathrm{GaAs}$ freeze out at this temperature. ${ }^{12}$

In the following we will describe the experimental data of Skuras et al.,$^{5}$ who used a sample with a doping slab having a thickness of $20 \AA$. This sample allows us to expect that the two-dimensional (2D) $D X$ model is a good approximation. ${ }^{10}$ A detailed comparison between experiment and theory will show the importance of correlation effects of charged impurities clearly, but good agreement can been obtained only for the lowest subband in the $d^{+} / D X^{-}$model. We think that the theoretical results for the higher subbands are overestimated because self-compensation occurs in the Si-doped layer. ${ }^{13,14}$ A simple estimation has been performed, which shows that inclusion of both self-compensation of the impurities and spatial correlations of charged impurities can explain the experimental data within both $D X$-center models. Therefore, in order to investigate the influence of $D X$ centers on the transport properties of $\delta$-doped GaAs effectively one has to use samples with relatively low doping density, so that mechanisms such as clustering and self-compensation can be neglected.

This paper is organized as follows. In Sec. II the quantum mobility calculation of the electrons in a $\delta$-doped structure is described, which shows clearly the importance of spatial correlations of charged impurities. The charge distribution in two dimensions is simulated by a Monte Carlo approach in Sec. III, where the pair-correlation functions and the structure factors are given in the effective scales. A detailed comparison of the theoretical results with the measured quantum mobilities is performed in Sec. VI. Our discussions and conclusions are presented in Sec. V.

\section{MOBILITY CALCULATIONS}

The structure under consideration, which was used by Skuras et al. ${ }^{5}$ is a ( $\mathrm{Si}$ ) slab-doped GaAs structure having a 2D doping concentration $N_{D}=1.1 \times 10^{13} \mathrm{~cm}^{-2}$ and a finite layer width $W_{D}=20 \AA$. The electronic structure of this system can be determined by a self-consistent calculation, ${ }^{15,10}$ which produces the energy $E_{I}$, the wave function $\psi_{I}(z)$, and the $2 \mathrm{D}$ electron density $N_{I}$ of the $I$ th subband as well as the Fermi energy $E_{F}$ and the total confinement potential $U_{\mathrm{EFF}}(z)$. In this calculation, influences of the background acceptors described by the $2 \mathrm{D}$ density $N_{A}$ and the thickness of the depletion layer $W_{A}\left(N_{A} / W_{A}=5 \times 10^{14} \mathrm{~cm}^{-3}\right)$, band nonparabolicity, and the exchange-correlation energy of the electrons have been taken into account. Therefore, the total electron energy is given by $E_{I}+\hbar^{2} k_{\|}^{2} / 2 m^{*}$, with $\vec{k}_{\|}$the electron wave vector in the $x-y$ plane that is parallel to the $\delta$ layer, and $m^{*}=m_{0}(1+\alpha P) /\left(1-\beta \hbar^{2} k_{F}^{2} / 2 m_{0}\right)$ the electron effective mass at the Fermi level, with $m_{0} / m_{e}=0.067$ the effective mass at the bottom of the conduction band of GaAs. Two coefficients, $\alpha=7.4 \times 10^{-3} \mathrm{kbar}^{-1}$ the pressure dependence and $\beta=1.07 \mathrm{eV}^{-1}$ the band nonparabolicity factor, are taken from Ref. 16.

With inclusion of the correlation effects of all the charged impurities described by the structure factor $s\left(q_{\|}\right)$in two dimensions, the quantum relaxation time, ${ }^{4,8}$ which is directly related to the quantum mobility for the electrons located at the $I$ th subband $\mu_{I}^{Q}=e \tau_{I}^{Q} / m^{*}$, can be expressed by

$$
\frac{1}{\tau_{I}^{Q}}=\frac{m^{*}}{\pi \hbar^{3}} \sum_{I^{\prime}} \int_{0}^{\pi} d \theta s\left(q_{\|}\right)\left|u_{I, I^{\prime}}\left(q_{\|}\right)\right|^{2},
$$

where $\left|u_{I, I^{\prime}}\left(q_{\|}\right)\right|^{2}$ is the square of the transition matrix element between state $\left|I, \vec{k}_{\|}\right\rangle$and state $\left|I^{\prime}, \vec{k}_{\|}^{\prime}\right\rangle$ due to the screened Coulomb potential expressed by

$$
\left|u_{I, I^{\prime}}\left(q_{\|}\right)\right|^{2}=\frac{4 \pi^{2} e^{4} N_{\text {c.i. }}}{q_{\|}^{2} W_{D}} \int_{-W_{D} / 2}^{W_{D} / 2} d Z_{i}\left|G_{I, I^{\prime}}\left(q_{\|}, Z_{i}\right)\right|^{2}
$$

where $N_{\text {c.i. }}$ is the $2 \mathrm{D}$ density of charged impurities in the $\delta$-doped layer, which equals $N_{d^{+}}+N_{A}$ for the $d^{+} / D X^{0}$ model and $N_{D}+N_{A}$ for the $d^{+} / D X^{-}$model. In Eq. (2),

$$
\begin{aligned}
G_{I, I^{\prime}}\left(q_{\|}, Z_{i}\right)= & \frac{1}{\epsilon_{0 J, J^{\prime}}} \epsilon_{\left(I, I^{\prime}\right),\left(J, J^{\prime}\right)}\left(\vec{q}_{\|}\right) \\
& \times \int_{-\infty}^{\infty} d z \psi_{J}(z) \psi_{J^{\prime}}(z) e^{-q_{\|}\left|z-Z_{i}\right|},
\end{aligned}
$$

with $\vec{q}_{\|}=\vec{k}_{\|}^{\prime}-\vec{k}_{\|}$the change in electron momentum due to charged-impurity scattering, and $\theta$ the angle between $\vec{k}_{\|}^{\prime}$ and $\vec{k}_{\|} ;$and $\epsilon_{0}$ the dielectric constant of GaAs and $\epsilon_{\left(I, I^{\prime}\right),\left(J, J^{\prime}\right)}^{-1}\left(\vec{q}_{\|}\right)$is the element of the inverse matrix of the dielectric function which will be calculated within the RPA. $^{17}$ 


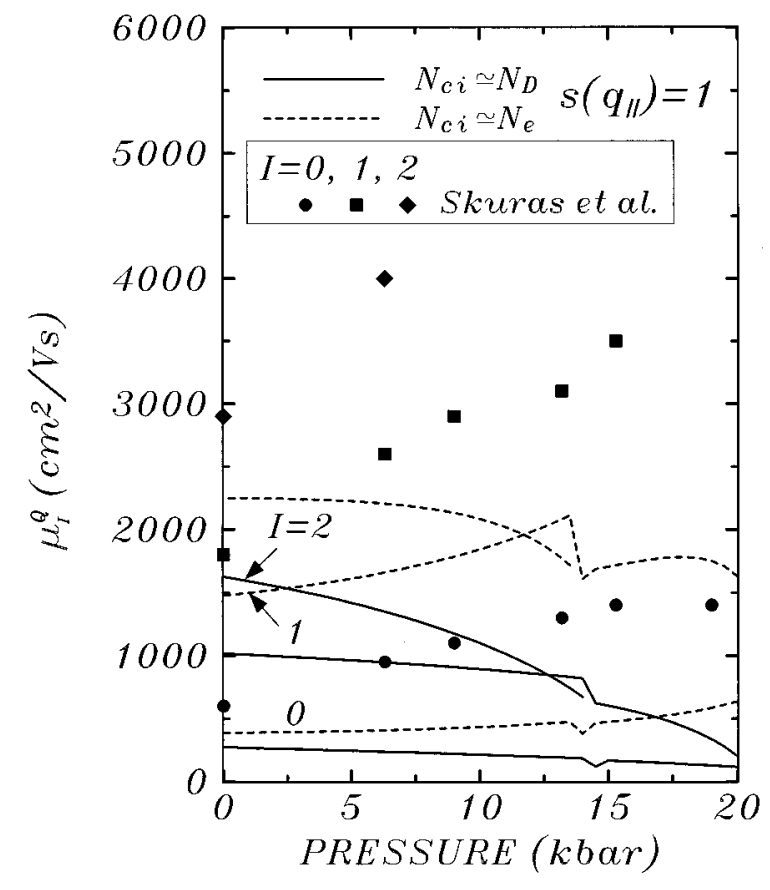

FIG. 1. The subband quantum mobility as a function of hydrostatic pressure for $\delta$-doped GaAs having a doping concentration $N_{D}=1.1 \times 10^{13} \mathrm{~cm}^{-2}$, a $\delta$-layer width $W_{D}=20 \AA$, and an acceptor concentration $N_{A}=0.5 \times 10^{12} \mathrm{~cm}^{-2}$. The symbols are experimental data in Ref. 5, and the curves theoretical results excluding the effects of $D X$ centers.

In the absence of the spatial correlations of the charged impurities, the value of the structure factor $s\left(q_{\|}\right)$is equal to 1. This describes a random distribution of the charges which is the same as that of the dopants. A theory for the mobility calculation in this case has been developed in Ref. 4, in which it was assumed that the density of the scatterers is equal to that of the free electrons $\left(N_{\text {c.i. }} \simeq N_{e}\right)$. This corresponds to the $d^{+} / D X^{0}$ model excluding the correlations. A similar numerical calculation of the mobility has been performed, the results of which are shown in Fig. 1 by the dashed curves for the pressure dependence of the quantum mobility of the electrons in the different subbands as compared to the experimental results (solid symbols) of Skuras et $a .^{5}$ It is clear that the existing theory seriously underestimates the electron mobility in such a structure, and even is not able to describe the measured data qualitatively. Furthermore, the calculated results obtained from the uncorrelated $d^{+} / D X^{-}$model $\left(N_{\text {c.i. }} \simeq N_{D}\right.$, solid curves $)$ cannot provide any improvement. As a consequence, one may expect that the structure factor $s\left(q_{\|}\right)$, i.e., the correlation effects of the charged impurities, should be responsible for the increase of the electron mobility under study.

It is well known that the $D X$ state will be more occupied with increasing doping concentration and/or the application of hydrostatic pressure. In the $d^{+} / D X^{-}$model the chargedimpurity density $N_{\text {c.i. }}$ remains constant, while it decreases in the $d^{+} / D X^{0}$ model with increasing pressure. This implies that the $d^{+} / D X^{0}$ model has an effect on the electron mobility through a decrease of the scatterer density, and the $d^{+} / D X^{-}$model does not. However, the structure factor $s\left(q_{\|}\right)$in Eq. (1) reflects the influence of the spatial correlations in the charge distribution in the $\delta$ layer, and diminishes the output of the integrals for both $D X$ models. Therefore, these correlation effects in the $\delta$-doped layer will lead to an increase in the electron mobility, as was shown in Refs. 6 and 8 for the bulk-doped structures.

\section{MONTE CARLO SIMULATIONS OF CORRELATION EFFECTS}

At certain conditions, part of the free electrons will be trapped by $D X$ centers. This results in the coexistence of two kinds $\left(d^{+}\right.$and $D X^{0}$ or $d^{+}$and $\left.D X^{-}\right)$of donor states, and reduces the total Coulomb energy of the system. If the temperature is higher than the freeze-out temperature, ${ }^{12}$ all $D X$-state electrons can move out of the donor centers. As a result, all impurities are positively charged in a random distribution. The structure factor, which influences the lowtemperature $(T=0 \mathrm{~K})$ electron mobility, is determined at $T=120 \mathrm{~K}$ for Si-doped GaAs. In present work we use a Yukawa potential to describe the interaction between any two $(i$ and $j$ ) impurities,

$$
U_{i, j}\left(\rho_{i, j}\right)=\frac{e_{i} e_{j}}{\epsilon_{0} \rho_{i, j}} \exp \left(-\frac{\rho_{i, j}}{\lambda}\right),
$$

where $e_{i}$ denotes the charge of the $i$ th center, $\rho_{i, j}$ the distance between the $i$ and $j$ donors, and $\lambda$ the electron screening length given by the semiclassical, three-dimensional Thomas-Fermi screening theory which has been proven to be a good approximation for the typical $\delta$-doped structures. ${ }^{18,10}$ The interaction depends weakly on the actual value of $\lambda$ for $\delta$-doped GaAs, which was determined to be $\lambda=50 \AA$ Afor two dimensions. ${ }^{18}$ This value has also been used in the present calculation.

The structure factor $s\left(q_{\|}\right)$results from the Fourier transformation of the pair-correlation functions which are different for two $D X$ models. In the $2 \mathrm{D} d^{+} / D X^{0}$ model, it is expressed by

$$
s\left(q_{\|}\right)=1-2 \pi N_{+} \int_{0}^{\infty}\left[1-g_{++}(\rho)\right] J_{0}\left(q_{\|} \rho\right) \rho d \rho
$$

where $N_{+}=N_{e}+N_{A}$ and the pair-correlation function $g_{++}(\rho)$ describes the probability of finding a positively charged impurity at a distance $\rho$ from a position at which there is already a given ionized donor; in the $2 \mathrm{D} d^{+} / D X^{-}$ model, it is given by

$$
\begin{aligned}
s\left(q_{\|}\right)= & 1-\frac{2 \pi}{N_{D}} \int_{0}^{\infty}\left[4\left(N_{e}+N_{A}\right)^{2}-g_{++}(\rho) N_{+}^{2}-g_{--}(\rho) N_{-}^{2}\right. \\
& \left.+2 g_{+-}(\rho) N_{+} N_{-}\right] J_{0}\left(q_{\|} \rho\right) \rho d \rho,
\end{aligned}
$$

where the three functions $g_{++}(\rho), g_{--}(\rho)$, and $g_{+-}(\rho)$ describe correlations between positive-positive, negativenegative, and positive-negative pairs, respectively, and $N_{ \pm}=\left(N_{D} \pm N_{e} \pm N_{A}\right) / 2$ are the densities of the \pm charges. 


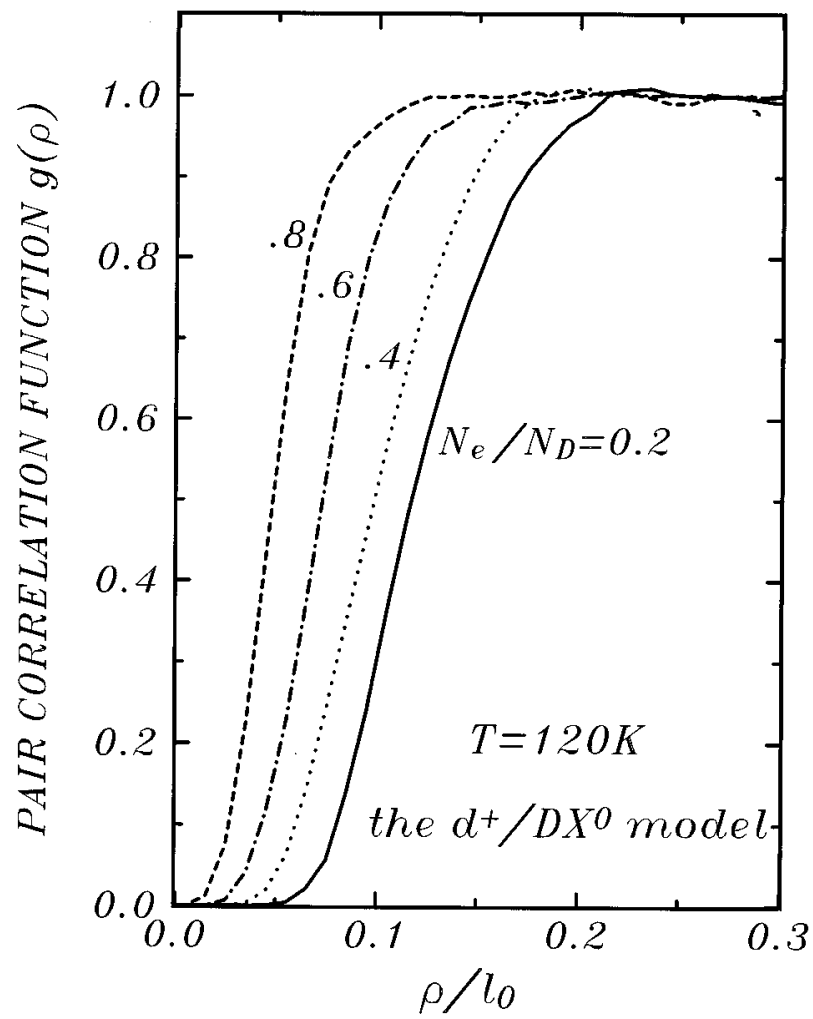

FIG. 2. Pair-correlation function in the $d^{+} / D X^{0}$ model obtained from Monte Carlo simulations at $T=120 \mathrm{~K}$ for $N_{+} / N_{D}=0.2,0.4$, 0.6 , and 0.8 .

A Monte Carlo calculation was developed in Ref. 9 to simulate the charge distribution in $\delta$-doped structures. Now we will extend this work to calculate the structure factor in order to obtain modifications of the mobility due to the correlation effects. The simulations use square geometry with periodic boundary conditions, in which the length of the square is 4 in units of length, and the total number of impurities is 1000 . This choice allows us to use the following conventional measurements: $l_{0}(\AA)=250 / n_{D}^{1 / 2}$ as the unit of length where $n_{D}\left(\mathrm{~cm}^{-2}\right)=N_{D} / 10^{13} ; \quad$ and $\quad R_{0}(\mathrm{meV})$ $=1162 / l_{0}$ as the unit of energy, so that the pair-correlation functions and the structure factors can been given in a universally functional form which depends on only the ratio of $N_{e} / N_{D}$. In general, the total free-electron density $N_{e}$ should be obtained by a solution of the equilibrium equation of the reservoirs of $D X$ centers and the free electrons. However, this work is very computer time consuming. In order to describe the measured electron mobility, one can take the experimental data of $N_{e}$ as input into the simulations, which displays the correlation effects in the structures.

Figure 2 shows the pair-correlation function $g(\rho)$ obtained from the Monte Carlo simulations for the $d^{+} / D X^{0}$ model at temperature $T=120 \mathrm{~K}$ for the four different ratios of $N_{e} / N_{D}=0.2,0.4,0.6$, and 0.8 , where we assume that $N_{A}$ is negligible. It is clear that the charges within the small separations are more correlated, that all the curves are ramplike rather than steplike, and that with increasing the value of $N_{e} / N_{D}$ the function becomes more steplike in character. This is consistent with the conclusion of Ref. 10 . We do not find any significant oscillations of $g(\rho)$ because the system under study is at high temperature. ${ }^{9}$

The three pair-correlation functions $\left[\right.$ (a) $g_{++}$, (b) $g_{--}$, and (c) $\left.g_{+-}\right]$in the $d^{+} / D X^{-}$model are plotted in Fig. 3 for $N_{e} / N_{D}=0.0,0.2,0.4,0.6$, and 0.8 . In this model the correlations are shorter ranged as compared with those in the $d^{+} / D X^{0}$ model, because the positive and negative charges always try to construct the closest pairs [see (c)] and diminish the importance of long-range correlations.

The structure factor $s\left(q_{\|}\right)$for the systems discussed in Figs. 2 and 3 is plotted in Fig. 4 within the $d^{+} / D X^{0}$ model (a) and the $d^{+} / D X^{-}$model (b). Notice the following: (1) The values of $s\left(q_{\|}\right)$obtained from the $d^{+} / D X^{0}$ model are systematically higher than those from the $d^{+} / D X^{-}$model because of the stronger correlations in the latter model. (2) At $q_{\|}=0$ the $d^{+} / D X^{-}$model gives a monotonously decreasing function for the structure factor with decreasing $N_{e} / N_{D}$, because decreasing $N_{e}$ implies increasing the number of the pairs of the positive and negative charges, which result in the stronger correlations in the system. This is different from the $d^{+} / D X^{0}$ model, where the structure factor

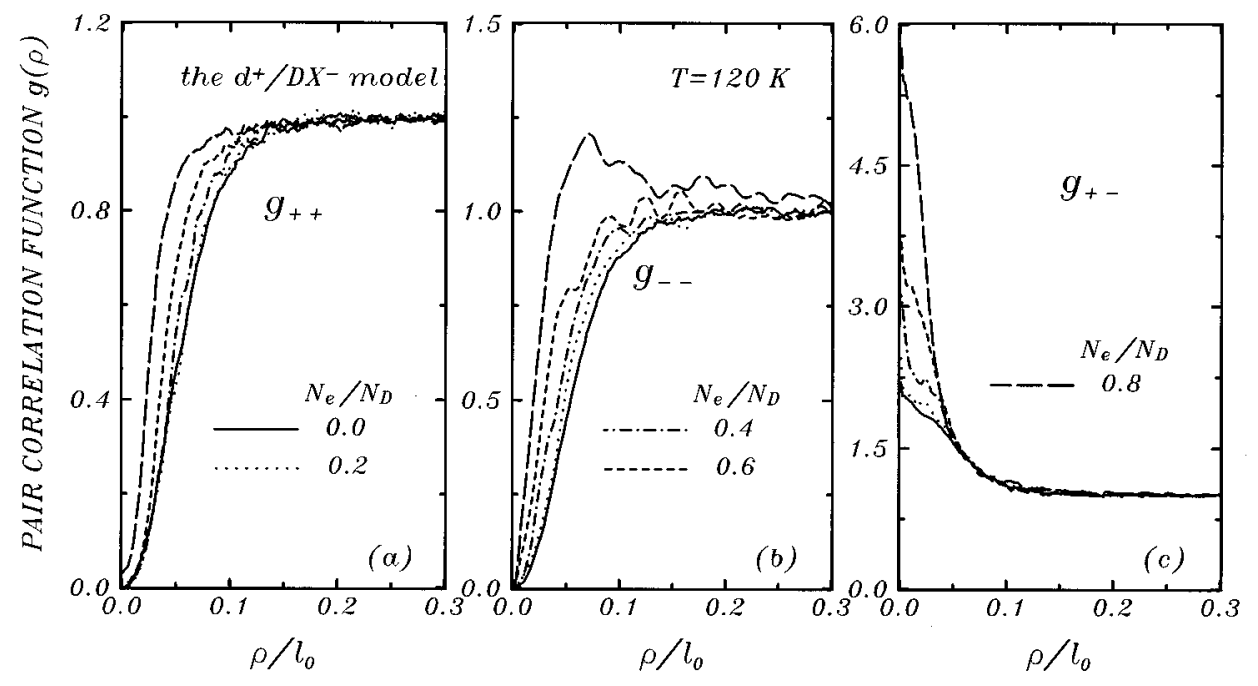

FIG. 3. The same as shown in Fig. 2 but now for three paircorrelation functions in the $d^{+} / D X^{-} \quad$ model including $N_{+} / N_{D}=0.0$ : (a) $g_{++}$, (b) $g_{--}$, and (c) $g_{+-}$. 

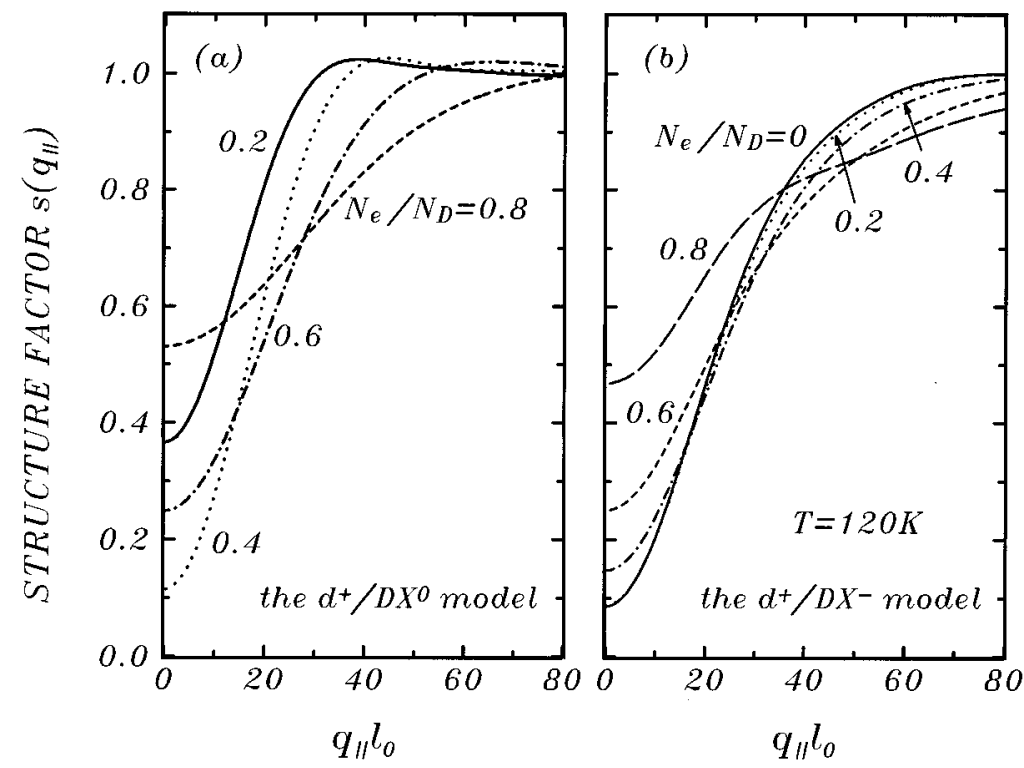

FIG. 4. The structure factor of the charge distribution obtained from the Fourier transforms of the pair-correlation functions given by Figs. 2 and 3. (a) is for the $d^{+} / D X^{0}$ model, and (b) for the $d^{+} / D X^{-}$model. for $N_{e} / N_{D}=0.2$ is close to that for $N_{e} / N_{D}=0.8$. This is due to the fact that $N_{e} / N_{D}=0$ and 1 for this model give no correlation effects, since there is only one kind of impurities at these two limits. (3) All the results are positive, and approach 1 with increasing momentum.

\section{COMPARISON WITH EXPERIMENT}

Using Shubnikov-de Haas and persistent photoconductivity measurements, Skuras et al. $^{5}$ obtained the hydrostaticpressure dependence of the free-electron densities and the electron quantum mobilities in the individual subbands of $\delta$-doped GaAs structures with $N_{D}=1.1 \times 10^{13} \mathrm{~cm}^{-2}$ and $W_{D}=20,50$, and $100 \AA$, respectively. The present work will be confined to the sample having $W_{D}=20 \AA$, since our correlation models are described in two dimensions.

Starting from a solution of the equilibrium equation of the reservoirs of $D X$ centers and the free electrons, ${ }^{10}$ we calcu- lated the electronic structure of the sample which was used by Skuras et al. with inclusion of spatial correlation effects of charged impurities in the $d^{+} / D X^{-}$and $d^{+} / D X^{0}$ models, where the pair-correlation functions were described by a step function in a short-range interaction model. Good agreement is found between theory and experiment for the electron density of each subband within both of the $D X$ models when all dopant atoms can act as $D X$ centers, in which two of the parameters of the $D X$ level were fitted: one is $d E_{D X} / d P=-9 \mathrm{meV} / \mathrm{kbar}$ for both models, and the other $E_{D X}=290 \mathrm{meV}$ at $P=0$ for $D X^{-}$and $E_{D X}=210 \mathrm{meV}$ for $D X^{0}$. However, all of these values are in the region of reported values. ${ }^{7,14}$ Therefore, further investigation is needed to test which $D X$ model is better to describe the experimental findings.

In Fig. 5 we show a comparison of the same measured mobilities (solid symbols) as those in Fig. 1 with the theoretical analysis within the two different $D X$ models including the correlation effects $\left[(\mathrm{a}) d^{+} / D X^{-}\right.$and (b) $\left.d^{+} / D X^{0}\right]$,

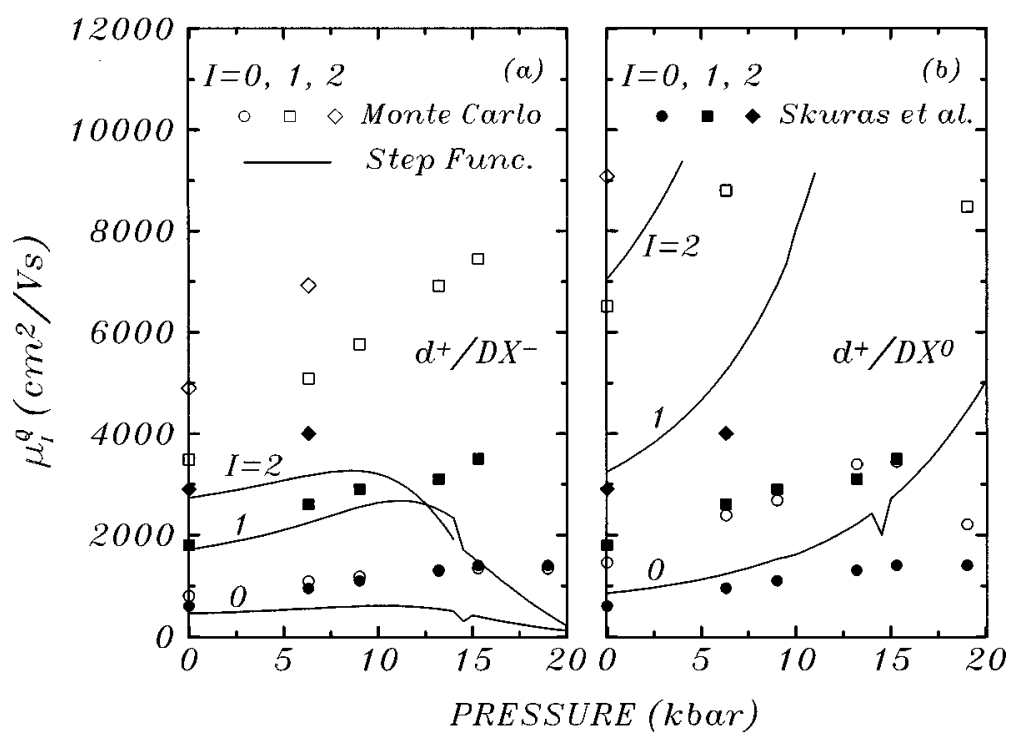

FIG. 5. Comparison of the measured quantum mobility in Ref. 5 (solid symbols) with the calculated results including the correlation effects within the step-function approximation (curves) and by the Monte Carlo simulations (open symbols) in the $d^{+} / D X^{-}$(a) and $d^{+} / D X^{0}$ (b) models. 


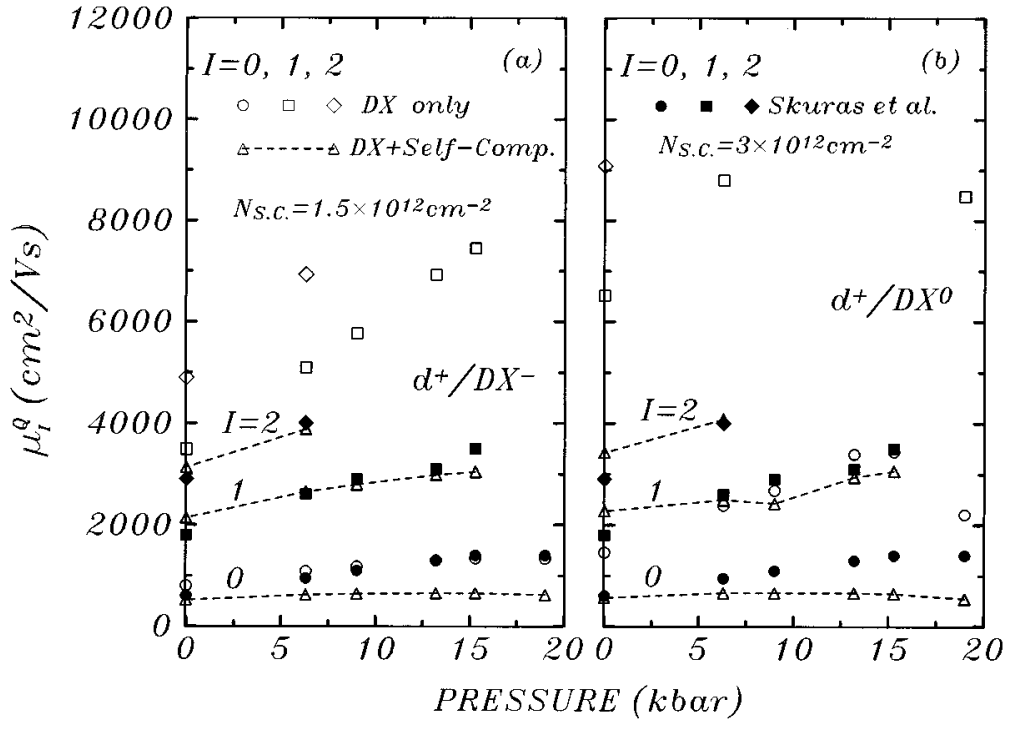

FIG. 6. Comparison of the measured quantum mobility in Ref. 5 (solid symbols) with the calculated results including only the correlation effects by the Monte Carlo simulations (open symbols), and with the results estimated from the $D X$ center and self-compensation mechanisms (triangles connected with dashed curves) in the $d^{+} / D X^{-}$ (a) and $d^{+} / D X^{0}$ (b) models. through Monte Carlo simulations (open symbols) as well as in the step-function approximation ${ }^{7,19}$ (curves). In the Monte Carlo calculation the experimental data of $N_{e}=7.2,6.02,5.45,4.36,3.87$, and 1.94 in units of $10^{12} \mathrm{~cm}^{-2}$ have been used which were obtained at the pressure $P=0.0,6.3,9.0,13.2,15.3$, and 19.0 in units of kbar, respectively. ${ }^{5}$ The mobility calculation is performed at zero temperature due to the experimental condition, but the structure factor is fixed at $T=120 \mathrm{~T}$, at which the $D X$ centers are frozen out. It is clearly shown that the correlation effects of the charged impurities enhance the electron mobility greatly. Note the following: (1) The $d^{+} / D X^{0}$ model gives larger corrections to the electron mobility than the $d^{+} / D X^{-}$model. This is due to the fact that the $d^{+} / D X^{0}$ model influences the mobility not only by the structure factor but also by a decrease of the density of charged impurities. (2) The $d^{+} / D X^{0}$ model overestimates systematically the quantum mobility, while the $d^{+} / D X^{-}$model, including correlation effects in the step-function approximation, improves the results at low pressure $P<10 \mathrm{kbar}$, especially at $P=0$ as compared to the theory excluding these effects (see Fig. 1). However it still underestimates the quantum mobility, and fails to describe the experimental findings in the highpressure region. (3) Monte Carlo simulations within the $d^{+} / D X^{-}$model give a good agreement for the electron mobility in the lowest subband for the whole pressure range, and for excited subbands they show a qualitative behavior, but the absolute values are twice as large as the measured data. A possible explanation for the latter discrepancy is deferred to Sec. V.

\section{DISCUSSIONS AND CONCLUSIONS}

We performed a theoretical study of the electron quantum mobility in the individual subbands of a $\delta$-doped GaAs structure, where the intrasubband and intersubband scatterings are considered within the electron-electron screening in the RPA. In order to improve upon previous calculations, a Monte Carlo approach was developed to simulate the charged-impurity distribution and to obtain the structure factor of this distribution which influences the electronic scattering directly. The importance of spatial correlations of charged impurities, which enhance the electron mobility greatly, has been shown. However, good agreement has been achieved between our calculation and experiment only for the lowest subband when the $d^{+} / D X^{-}$model is used. This cannot provide definite proof to confirm which model is better to describe the electronic properties of $D X$ centers in the present structure.

In order to explain the limiting electron density in GaAs with high Si-doping concentrations, several possible mechanisms $^{13,14}$ have been proposed, such as selfcompensation of $\mathrm{Si}$ atoms, which should also influence the electron mobility. Including this mechanism into the present theory one can calculate the electron mobility through a fitting of the density of self-compensation $\mathrm{Si}$ atoms $\left(N_{\mathrm{S} . \mathrm{C} .}\right)$, which are supposed to be in a random distribution in the $\delta$ layer. The maximum value of $N_{\text {S.C. }}$ must be smaller than the difference between the doping concentration and the total free-electron density at ambient pressure. For the sample under investigation this means that one has $0<N_{\text {S.C. }}<3.8 \times 10^{12} \mathrm{~cm}^{-2}$. If we neglect the influence of self-compensation on the distribution of all other charged impurities in which the spatial correlations occur, the total electron mobility due to different mechanisms (e.g., $D X$ centers and self-compensation atoms) can be approximated by

$$
\frac{1}{\mu_{\mathrm{tot}}}=\frac{1}{\mu_{D X}}+\frac{1}{\mu_{\mathrm{S} . \mathrm{C} .}} .
$$

Figure 6 shows the calculated mobility including selfcompensation given by the triangles connected by the dashed curves as compared with the experimental data (solid symbols) and the results from the previous calculation shown in Fig. 5 (open symbols). A considerable improvement is obtained for both models by use of the different values of $N_{\text {S.C. }}: 1.5 \times 10^{12} \mathrm{~cm}^{-2}$ for the $d^{+} / D X^{-}$model and 
$3.0 \times 10^{12} \mathrm{~cm}^{-2}$ for the $d^{+} / D X^{0}$ model. It is clearly shown that both $D X$ models can give almost the same results, which are in reasonable agreement with the experiment except for the lowest subband at high pressures.

The present analysis proves that it is impossible to determine clearly the electronic properties of $D X$ centers in samples with such a high doping concentration, ${ }^{5,14}$ since selfcompensation cannot be ruled out. In order to clarify this problem effectively we propose to investigate our recently proposed $\delta$-doped quantum barrier structures, ${ }^{10,19}$ which need lower doping concentrations to populate the $D X$ state; thus the effects of other mechanisms (self-compensation, clustering and so on) may be expected to be negligible.

\section{ACKNOWLEDGMENTS}

One of us (J.M.S.) was supported by the FUNCAP (Brazil), and F.M.P. by the Belgian National Science Foundation. J.T.D. acknowledges support by the Belgian National Science Foundation (NFWO, No. G.0287.95). Part of this work was performed by the Phantoms Network of Excellence ESPRIT-III BRA Action 7360.
${ }^{1}$ See, e.g., Delta Doping of Semiconductors, edited by E. F. Schubert (Cambridge University Press, London, 1995).

${ }^{2}$ P. M. Mooney, J. Appl. Phys. 67, R1 (1990).

${ }^{3}$ P. M. Koenraad, A. F. W. van de Stadt, J. M. Shi, G. Q. Hai, N. Studart, P. Vansant, F. M. Peeters, J. T. Devreese, J. A. A. J. Perenboom, and J. H. Wolter, Physica B 211, 462 (1991).

${ }^{4}$ G. Q. Hai, N. Studart, and F. M. Peeters, Phys. Rev. B 52, 8363 (1995).

${ }^{5}$ E. Skuras, R. Kumar, R. L. Williams, R. A. Stradling, J. E. Dmochowski, E. A. Johnson, A. Mackinnon, J. J. Harris, R. B. Beall, C. Skierbeszewski, J. Singleton, P. J. van der Wel, and P. Wisniewski, Semicond. Sci. Technol. 6, 535 (1991).

${ }^{6}$ E. P. O’Reilly, Appl. Phys. Lett. 55, 1409 (1989).

${ }^{7}$ J. Kossut, Z. Wilamowski, T. Dietl, and K. Światek, Acta Phys. Pol. A 79, 49 (1991).

${ }^{8}$ P. J. van der Wel, M. J. Anders, L. J. Giling, and J. Kossut, Semicond. Sci. Technol. 8, 211 (1993).

${ }^{9}$ P. Sobkowicz, Z. Wilamowski, and J. Kossut, Semicond. Sci. Technol. 7, 1155 (1992).

${ }^{10}$ J. M. Shi, P. M. Koenraad, A. F. W. van de Stadt, F. M. Peeters,
J. T. Devreese, and J. H. Wolter, Phys. Rev. B 54, 7996 (1996).

${ }^{11}$ T. N. Morgan, J. Electron. Mater. 20, 63 (1991).

${ }^{12}$ R. Piotrzkowski, T. Suski, P. Wiśniewski, K. Ploog, and J. Knecht, J. Appl. Phys. 68, 3377 (1990).

${ }^{13}$ E. F. Schubert, R. F. Kopf, J. M. Kuo, H. S. Luftman, and P. A. Garbinski, Appl. Phys. Lett. 57, 497 (1990).

${ }^{14}$ P. M. Koenraad, W. de Lange, F. A. P. Blom, M. R. Leys, J. A. A. J. Perenboom, J. Singleton, and J. H. Wolter, Semicond. Sci. Technol. 6, B143 (1991).

${ }^{15}$ M. H. Degani, Phys. Rev. B 44, 5580 (1991).

${ }^{16}$ D. K. Maude, J. C. Portal. L. Dmowski, T. Foster, L. Eaves, M. Nathan, M. Heiblum, J. J. Harris, and R. B. Beall, Phys. Rev. Lett. 59, 815 (1987).

${ }^{17}$ L. R. González, J. Krupski, and T. Szwacka, Phys. Rev. B 49, 11111 (1994).

${ }^{18}$ P. Sobkowicz, Z. Wilamowski, and J. Kossut, J. Phys. Condens. Matter 5, 5283 (1993).

${ }^{19}$ P. M. Koenraad, J. M. Shi, A. F. W. van de Stadt, A. Smets, J. A. A. J. Perenboom, F. M. Peeters, J. T. Devreese, and J. H. Wolter, Superlatt. Microstruct. (to be published). 